\title{
Schiff Base Dinuclear Zn(II) Complexes as Assisting Fluorescent Probe for CD19 Antibody Protein
}

\author{
Sae Yasuda, Masahiro Takase, Atsuko Sano, and Takashiro Akitsu
}

\begin{abstract}
In immunity functional tests with flow cytometry, proteins emerging surface or inner part of lymphocytes are analyzed with the aid of fluorescent emission generally. In this study, we employed newly prepared Schiff base binuclear Zn(II) complexes instead of commercially available fluorescent labeling agents to improve intensity of fluorescence. According to UV-vis, fluorescent, and flow cytometry measurements, $\mathrm{X}$-ray crystallography, and in-silico docking simulation (GOLD), it can remarkably enhance fluorescent intensity for CD19 antibody without specific binding to CD19.
\end{abstract}

Index Terms-Zinc(II) complex, schiff base, CD19, fluorescent, flow cytometry.

\section{INTRODUCTION}

Lymphocytes include T cells, B cells, and NK cells. They are major cellular components for adaptive immune systems. Primary immunodeficiency diseases (PIDs) are rare genetic diseases characterized by abnormal types of lymphocytes. Detection of surface markers on lymphocytes of patients by flow cytometry (FCM) is one of useful tools to diagnosis of PIDs conventionally [1], [2].

Flow cytometry can be used to assess not only expression of cell-surface proteins, but also intracellular molecules. However, intracellular protein analysis is often difficult due to technical reasons. The fluorescent conjugated to antibodies for intracellular proteins is often weaker than that conjugated to antibodies for cell-surface proteins. Loss of fluorescent emission or dissociation from the protein molecules must be a part of the reasons.

In this context, we have studied on application of (chiral) Schiff base metal complexes and their hybrid systems as fluorescence probes. Three systems were constructed for quantitative fluorescence sensing of metal ions by using chiral Schiff base zinc(II) complexes. The organic-inorganic composite materials made from chiral ones and spiropyran, which may change in fluorescence intensity of the complexes due to the photoisomerization of the ring opening type merocyanine closed ring type spiropyran. It has been fabricated. They composed of a trans-type chiral Schiff base zinc(II) complexes and merocyanine, which is open-form of spiropyran after photoisomerization. Depending on intermolecular interactions and quenching, increase $\left(\mathrm{Zn}^{2+}\right)$ or decrease $\left(\mathrm{Cu}^{2+}\right.$ and $\left.\mathrm{Gd}^{3+}\right)$ of fluorescence intensity of zinc(II) complexes could be observed as functions of concentration

Manuscript received February 9, 2017; revised June 5, 2017.

The authors are with the Department of Chemistry, Faculty of Science, Tokyo University of Science, Tokyo 162-8601, Japan (e-mail: akitsu@rs.kagu.tus.ac.jp). of metal ions [3].

Moreover, in hybrid or composite systems with lysozyme protein, we subjected to UV, fluorescence, and CD spectroscopy [4]. The strongest intensity result of the absorption and fluorescence spectra stepwise change the concentration of, in the absorption spectrum increases and the shift of the peak due to the increased concentration of lysozyme was observed. Extinction due to the complex concentration increase could be observed in the fluorescence spectra. Than the energy transfers between the complex and the lysozyme this has occurred, the presence of molecular interactions between metal complex and protein molecules has been suggested.

Herein, we have prepared novel new Schiff base dinculear zinc(II) complex ( $\mathbf{Z n}_{2} \mathbf{L}$, Scheme 1) as a fluorescence probe. We will discuss its potentially application for the use of medical studies e.g. FCM, beyond conventional fluorescence quenching, by docking to CD19 antibody protein.

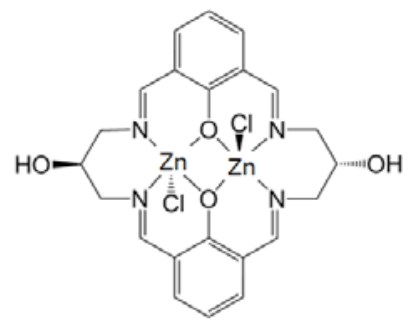

Scheme 1. Zn(II) compleX $\mathbf{Z n}_{2} \mathbf{L}$.

\section{EXPERIMENTAL SECTION}

\section{A. General Procedures}

Chemicals of the highest commercial grade available purchased from Wako and Kanto-Kagaku were used as received without further purification. 2, 6-dimethylphenol was synthesized according to the literature procedure [5], [6]. Commercially available non-labelling antibody purified mouse anti-human CD19 (BD 555410) were used.

\section{B. Preparations}

Saturated methanol solution of 1,3-diamino-2-propanol (0.018 g, $0.2 \mathrm{mmol})$ and $\mathrm{ZnCl}_{2}(0.027 \mathrm{~g}, 0.2 \mathrm{mmol})$ was prepared and 2,6-difolmylphenol $(0.030 \mathrm{~g}, 0.2 \mathrm{mmol})$ dissolved in methanol was added and refluxed for $4 \mathrm{~h}$ and yellow powder was obtained, were filtered and washed with cold methanol. Single crystals suitable for X-ray crystallography could be obtained when water solution was evaporated for 2 months. Yield: 50.0\% IR (KBr): 1638 (s) $(\mathrm{C}=\mathrm{N}),{ }^{1} \mathrm{H}-\mathrm{NMR}\left(\mathrm{DMSO}-\mathrm{d}^{6}, 300 \mathrm{MHz}\right): \delta 3.813(\mathrm{~m}, 4 \mathrm{H})$, 4.100 (6H, q), 5.216 (d, 1H), 5.355 (d, 1H), 6.938 (t, 2H), 
7.656 (q, 4H), 8.458 (d, 4H). Anal. Found C; 41.98, H; 3.22, $\mathrm{N}$; 8.63. Calc. $\mathrm{C} ; 42.20, \mathrm{H} ; 3.86, \mathrm{~N} ; 8.95 \%$ for $\mathrm{C}_{22} \mathrm{H}_{22} \mathrm{Cl}_{2} \mathrm{Zn}_{2} \mathrm{~N}_{4} \mathrm{O}_{4}\left(\mathrm{H}_{2} \mathrm{O}\right)$.

\section{Physical Measurements}

Elemental analyses (C, H, N) were carried out with a Perkin-Elmer 2400II CHNS/O analyzer at Tokyo University of Science. IR spectra were recorded on a JASCO FT-IR 4200 spectrophotometer in the range of $4000-400 \mathrm{~cm}^{-1}$ at 298 K. Electronic spectra were measured on a JASCO V-570 spectrophotometer in the range of 900-200 nm at $298 \mathrm{~K}$ and a JASCO V-650 spectrophotometer equipped with polarizer in the range of $800-220 \mathrm{~nm}$ at $298 \mathrm{~K}$. Fluorescence spectra were measured on a JASCO FP-6200 spectrophotometer in the range of $720-220 \mathrm{~nm} .{ }^{1} \mathrm{H}-\mathrm{NMR}$ spectra were recorded on a JEOL JMN-300 spectrometer (300 MHz). Flow Cytometry (FCM) was carried out with a BD LSRFFortessa cell analyzer by staining cells.

\section{X-ray Crystallography}

Deep greenish prismatic single crystals of $\mathbf{Z} \mathbf{n}_{\mathbf{2}} \mathbf{L}$ were glued on top of a glass fiber and coated with a thin layer of epoxy resin to measure the diffraction data. Intensity data were collected on a Bruker APEX2 CCD diffractometer with graphite monochromated $\mathrm{MoK} \alpha$ radiation $(\lambda=0.71073 \AA)$. Data analysis was carried out with a SAINT program package [7]. The structures were solved by direct methods with a SHELXS-97 and expanded by Fourier techniques and refined by full-matrix least-squares methods based on $F^{2}$ using the program SHELXL-97 [8]. An empirical absorption correction was applied by a program SADABS [9]. All non-hydrogen atoms were readily located and refined by anisotropic thermal parameters. All hydrogen atoms were located at geometrically calculated positions and refined using riding models. Acetate ions may be disordered potentially according to large thermal factors. CCDC 1536386.

Crystallographic data for $\mathbf{Z n}_{2} \mathbf{L}: \mathrm{C}_{22} \mathrm{H}_{22} \mathrm{Cl}_{2} \mathrm{~N}_{4} \mathrm{O}_{6} \mathrm{Zn}_{2}$, Triclinic, $\quad P-1, \quad Z=1, \quad a=7.7952(12), \quad b=8.1658(12)$, $c=10.3171(16) \quad \AA, \quad \alpha=90.1266(16), \quad \beta=95.1280(18)$, $\gamma=95.1280(18), \quad V=597.78(16) \quad \AA^{3}, \quad \rho_{\text {calc }}=1.739 \mathrm{~g} / \mathrm{cm}^{3}$, $\mu=2.347 \mathrm{~mm}^{-1}, F(000)=324, S=2.063, R_{1}[\mathrm{I}>2 \sigma(\mathrm{I})]=0.0684$, $w R_{2}=0.2259$.

\section{E. Docking Simulation}

CCDC GOLD suite was used for protein-ligand docking simulation. Crystal structure of CD19 was obtained from PDB:1IGY.

\section{RESUlTS AND DISCUSSION}

\section{A. Crystal Structure of $\mathbf{Z n _ { 2 }} \mathbf{L}$}

As summarized in Fig. 1, each chromophore of a dinuclear complex $\mathbf{Z} \mathbf{n}_{2} \mathbf{L}$ affords a five-coordinated square pyramidal coordination geometry with chloride axial ligands towards opposite direction. All geometrical parameters were within common ranges for the analogous compounds [10].

\section{B. Spectra of $\mathbf{Z} \mathbf{n}_{2} \mathbf{L}$}

UV-vis spectra of $\mathbf{Z} \mathbf{n}_{2} \mathbf{L}$ (0.01, 0.02, 0.03, 0.04, and 0.05
$\mathrm{mM}$ phosphate-buffered saline (PBS) solutions) appeared at $210 \mathrm{~nm}\left(\varepsilon=33000 \mathrm{M}^{-1} \mathrm{~cm}^{-1}\right), 249 \mathrm{~nm}\left(\varepsilon=50000 \mathrm{M}^{-1} \mathrm{~cm}^{-1}\right)$, and $353 \mathrm{~nm}\left(\varepsilon=11000 \mathrm{M}^{-1} \mathrm{~cm}^{-1}\right)$, while diffuse reflectance spectra appeared at $393 \mathrm{~nm}$. Fluorescence spectra of $\mathbf{Z} \mathbf{n}_{\mathbf{2}} \mathbf{L}$ (0.01, 0.02, 0.03, 0.04, and $0.05 \mathrm{mM}$ phosphate-buffered saline (PBS) solutions) appeared at $\lambda_{e m}=410 \mathrm{~nm}\left(\lambda_{e x}=265\right)$, $\lambda_{e m}=410 \mathrm{~nm}\left(\lambda_{e x}=360 \mathrm{~nm}\right)$, and $\lambda_{e m}=510 \mathrm{~nm}\left(\lambda_{e x}=405 \mathrm{~nm}\right.$ corresponding to FCM violet laser) enhancing intensity with increasing concentration.

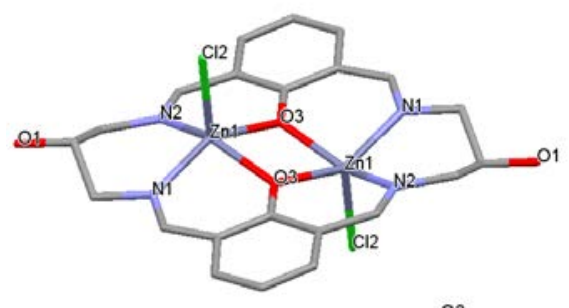

.02

Fig. 1. Crystal structure of $\mathbf{Z n}_{\mathbf{2}} \mathbf{L}$. showing selected atom labeling scheme. Hydrogen atoms and symmetry labels are omitted for clarity. Selected bond lengths. $[\AA]$ and angles [ [ ${ }^{\circ}$ : $\mathrm{Cl2}-\mathrm{Zn} 1=2.322(1), \mathrm{N} 1-\mathrm{Zn} 1=2.067(4), \mathrm{N} 2-\mathrm{Zn} 1$ = 2.078(4), O3-Zn1 = 2.061(3), O3-Zn1 = 2.077(3), Zn1-O3 = 2.077(3), $\mathrm{N} 1-\mathrm{Zn} 1=2.067(4), \mathrm{N} 2-\mathrm{Zn} 1=2.078(4), \mathrm{O} 3-\mathrm{Zn} 1=2.061(3), \mathrm{Cl} 2-\mathrm{Zn} 1=$ 2.322(1), Zn1-O3-Zn1 = 104.6(1), Cl2-Zn1-N1 = 104.9(1), Cl2-Zn1-N2 = 104.2(1), Cl2-Zn1-O3 = 105.08(9), Cl2-Zn1-O3 = 103.19(9), N1-Zn1-N2 = 93.4(2), N1-Zn1-O3 = 148.5(1), N1-Zn1-O3 = 88.6(1), N2-Zn1-O3 = 88.5(1), N2-Zn1-O3 = 151.0(1), O3-Zn1-O3 = 75.4(1), Zn1-O3-Zn1 = 104.6(1), C13-O3-Zn1 = 127.6(3), O3-Zn1-N1 = 88.6(1), O3-Zn1-N2 = 151.0(1), O3-Zn1-O3 = 75.4(1), O3-Zn1-Cl2 = 103.19(9), N1-Zn1-N2 = 93.4(2), N1-Zn1-O3 = 148.5(1), N1-Zn1-Cl2 = 104.9(1), N2-Zn1-O3 = 88.5(1), N2-Zn1-Cl2 = 104.2(1), O3-Zn1-Cl2 = 105.08(9).

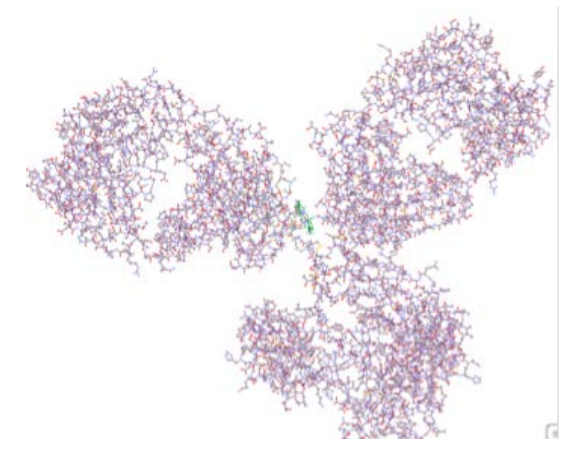

Fig. 2. Protein (CD19) - ligand $\left(\mathbf{Z n}_{2} \mathbf{L}\right)$ docking simulated with a GOLD program.

\section{Spectra of CD19+ $\mathbf{Z n} \mathbf{L}$}

Prior to spectral measurement, in-silico docking simulation was carried out to estimate where $\mathbf{Z n _ { 2 }} \mathbf{L}$ will be easily included in CD19 molecules. As depicted in Fig. 2 of the best score (40.7453), $\mathbf{Z} \mathbf{n}_{2} \mathbf{L}$ molecules may be included in the center of trimer structure of CD19 assemblies rather than in peptide chains (Fig. A1).

UV-vis spectra of non-labelling CD19(5 $\mu \mathrm{L})+\mathbf{Z n} \mathbf{n}_{2} \mathbf{L}(0.01$, $0.02,0.03,0.04$, and $0.05 \mathrm{mM}$ phosphate-buffered saline (PBS) solutions) appeared at $210 \mathrm{~nm}\left(\varepsilon=33000 \mathrm{M}^{-1} \mathrm{~cm}^{-1}\right)$, $249 \mathrm{~nm}\left(\varepsilon=50000 \mathrm{M}^{-1} \mathrm{~cm}^{-1}\right)$, and $353 \mathrm{~nm}(\varepsilon=11000$ $\left.\mathrm{M}^{-1} \mathrm{~cm}^{-1}\right)$, which were almost similar to those of $\mathbf{Z} \mathbf{n}_{\mathbf{2}} \mathbf{L}$ only. As shown in Fig. 3, fluorescence spectra of $\mathbf{Z} \mathbf{n}_{2} \mathbf{L}$ (0.01, 0.02, $0.03,0.04$, and $0.05 \mathrm{mM}$ phosphate-buffered saline (PBS) solutions) appeared at $\lambda_{e m}=410 \mathrm{~nm}\left(\lambda_{e x}=265\right), \lambda_{e m}=410 \mathrm{~nm}$ $\left(\lambda_{e x}=360 \mathrm{~nm}\right)$, and $\lambda_{e m}=380$ and $510 \mathrm{~nm}\left(\lambda_{e x}=405 \mathrm{~nm}\right.$ 
corresponding to FCM violet laser). In all cases, quenching by docking to CD19 could not be observed. Moreover, emission intensity of $\mathbf{Z} \mathbf{n}_{\mathbf{2}} \mathbf{L}$ was gained after docking to CD19, which suggested appropriate including to CD19.

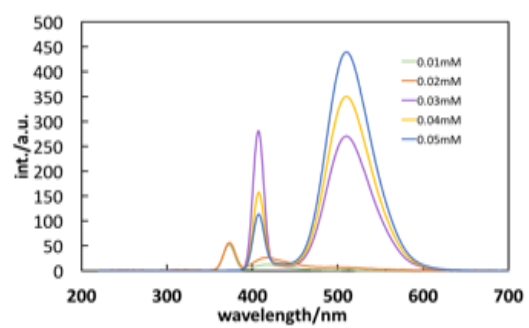

Fig. 3. Concentration (0.01-0.05 mM) dependence of fluorescence spectra of $\mathrm{CD} 19+\mathbf{Z n} \mathbf{n}_{2} \mathbf{L}\left(\lambda_{e x}=405 \mathrm{~nm}\right)$.
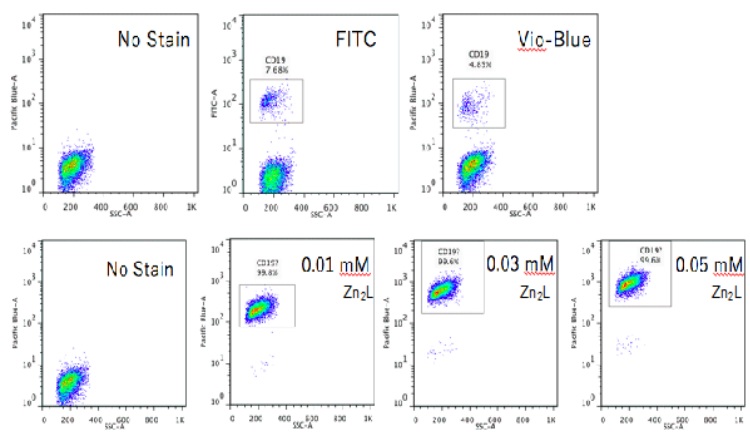

Fig. 3. FCM of several conditions.

\section{FCM of CD19+Zn $\mathbf{n}_{2} \mathbf{L}$}

In order to finding appropriate dyes and conditions, six dyes of labelling antibody (FITC, PE, ECD, PC7, APC, and Vio-Blue) was tested by means of surface staining, staining after cells fixation, and after cell permeabillization. Among these dyes, FITC and Vio-Blue were better than other ones. Then, $\mathbf{Z n} \mathbf{2} \mathbf{L}$ (0.01, 0.03, and 0.05 mM), FITC, and Vio-Blue were compared under the three conditions of treatments. In all results of FCM, as exhibited in Fig. 3 (Figs. A2-4), $\mathbf{Z} \mathbf{n}_{2} \mathbf{L}$ could increase emission intensity $\left(\lambda_{e x}=405 \mathrm{~nm}\right)$, though every parts of antibody were stained because of lack of specificity of $\mathbf{Z} \mathbf{n}_{2} \mathbf{L}$ in docking to void space of CD19, which was suggested in Fig. 2.

\section{CONCLUSION}

A newly prepared Schiff base dinuclear $\mathrm{Zn}$ (II) complex play a role in gaining emission intensity for FCM of CD19 antibody, though specificity of docking to CD19 could not work at present.

Indeed, biological or medical Zn(II) ions and their fluorescent detection will be become important. Recently $\mathrm{Zn}(\mathrm{II})$ ion have a wide range of different roles in immunity such as the second messenger in signal transduction. Zn(II) ion is an inhibited phosphatase PTEN, enhances the phosphorylation of AKT by interleukin 2 (IL2). Therefore, went and put a stimulus to the cells in a state in which a certain amount fluorescence by the addition of $\mathrm{Zn}$ (II) complex was out fluorescence disappears, and external field stimulation sex fluorescent probe has been sought as a medical research tool. This is either $\mathrm{Zn}$ (II) complex that has been made is to work in the same way as zinc, range of applications will change depending on whether there is an inhibitory effect as of pyrithione [11], [12].

By the way, besides normally experimental approaches, computational approaches (in-silico simulation like this study) may be valid not only for interpretation of experiments but also prediction or intentional design of hybrid functional materials composed of metal complexes. Relatively, compared with our previous studies of analogous topics, interpretation of experimentally spectral data and discussion of optimized structures of the complexes were successfully supported by TD-DFT computational data. For example, some electronic functions, for example current density of electron transfer from cathodes to a metalloenzyme via a mediator complex depends on molecular orientation clearly. Thus we will furthermore discuss to compare with the experimental facts and some theoretical frameworks towards theoretical interpretation of them or innovative material design of new systems organic/inorganic hybrid including chiral Schiff base metal complexes [13].

\section{APPENDIX}

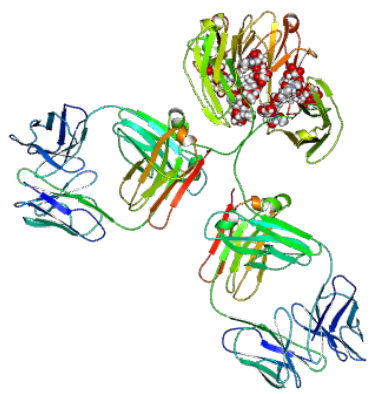

Fig. A1. Proposed (bad scores) protein (CD19) - ligand $\left(\mathbf{Z n}_{\mathbf{2}} \mathbf{L}\right)$ docking feature in peptide chains.

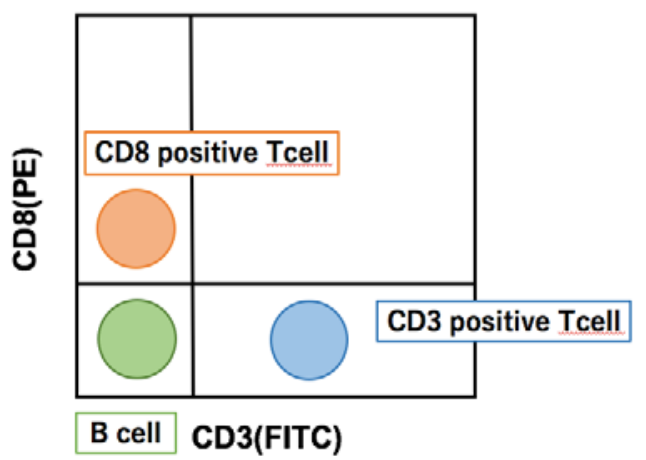

Fig. A2. Interpretation of FCM peaks.

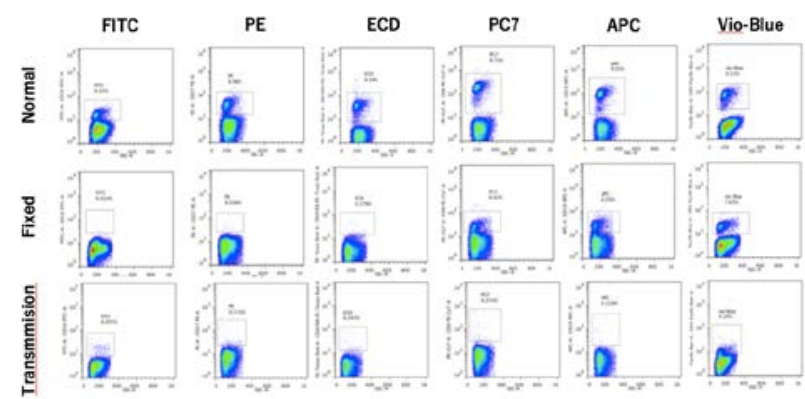

Fig. A3. FCM of several dyes. 


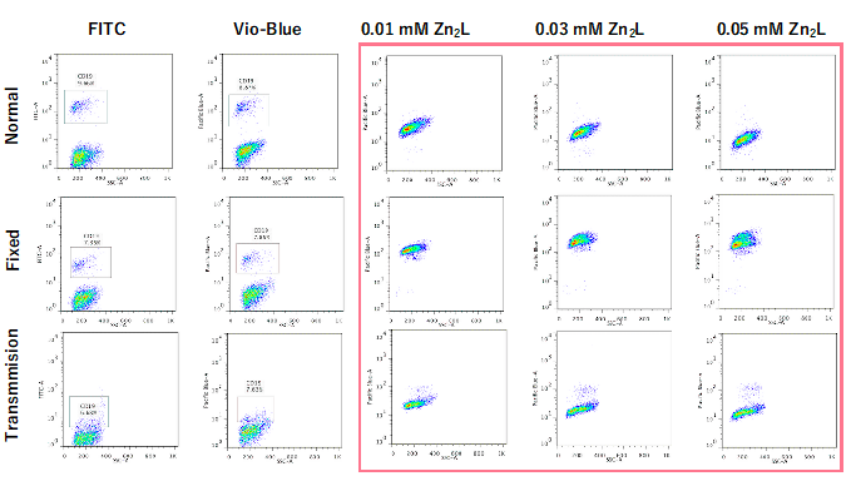

Fig. A4. FCM of comparison of $\mathbf{Z n}_{2} \mathbf{L}$ (red box) and better two dyes.

\section{ACKNOWLEDGMENT}

The authors thank Prof. Kohsuke Imai (Department of Pediatrics and Developmental Biology Tokyo Medical and Dental University) for the use of FCM instruments.

\section{REFERENCES}

[1] C. Orieji, "Current applications of flow cytometry in the diagnosis of primary immunodeficiency diseases,” Arch. Pathol. Lab. Med., vol. 128, pp. 23-31, January 2004.

[2] A. Durandy, S. Kracker, and A. Fisch, Nat. Rev. Immunol., vol. 13, pp. 519-533, June 2013.

[3] M. Aonuma, T. Onodera, S. Morito, A. Wada, and T. Akitsu, "Sensing of metal ions by hybrid systems of a chiral Schiff base Zn(II) complex and spiropyran,” J. Appl. Sol. Chem. Model., vol. 2, pp. 96-104, May 2013.

[4] T. Hayashi and T. Akitsu, "Threonine: Food sources, functions and health benefits,” USA, Nova Science Publishers, Inc., ch. 3, pp. 49-72, June 2015.

[5] M. Mihara, T. Nakai, T. Iwai, T. Ito, T. Ohno, and T. Mizuno, "Solvent-free iron(III) chloride catalyzed O-, S-, and N-Acylation under mild conditions,” Synlett, vol. 2, pp. 253-255, February 2010.
[6] P. Lin, M. Lecl`ere, J. Long, T. J. Burchell, I. Korobkov, R. Cl’erac, and M. Murugesu, "Fluorescent dialdehyde ligand for the encapsulation of dinuclear luminescent lanthanide complexes," Dalton Trans., vol. 39, pp. 5698-5704, May 2010.

[7] Bruker, SMART and SAINT, Madison, Bruker AXS Inc.: Wisconsin, 1998.

[8] G. M. Sheldrick, “A short history of SHELX,” Acta Crystallogr A., vol. 64, pp. 112-122, September 2008.

[9] G. M. Sheldrick, "SADABS program for empirical absorption," Correction of Area Detector Data: University of Gottingen, 1996.

[10] M. Takase and T. Akitsu, Polymer Science Book Series, Formatex Research Center, Spain, vol. 1, pp. 301-308, July 2016.

[11] H. Haase and L. Rink, "Multiple impacts of zinc on immune function," Metallomics, vol. 6, pp. 1175-1180, July 2014.

[12] E. Heiliger, A. Osmanagic, H. Haase, N. Golenhofen, A. M Grabrucker, A. Weth, and W. Baumgartner, “ $N$-cadherin-mediated cell adhesion is regulated by extracellular $\mathrm{Zn}^{2+}$,” Metallomics. vol. 7, pp. 1277-1287, July 2015.

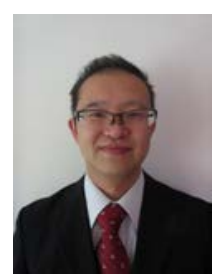

Takashiro Akitsu was born in Hamamatsu city, Japan, in October 1971. He graduated from the Department of Chemistry, Graduate School of Science, Osaka University (Toyonaka city, Osaka, Japan) and got his $\mathrm{PhD}$ degree in 2000 in the field of physical and inorganic chemistry, especially coordination chemistry.

He was appointed as a postdoctoral fellow at Osaka University, an assistant professor at Keio University, visiting scholar at Stanford University, and junior associate professor, associate professor, and full professor (since 2016) at the Department of Chemistry, Faculty of Science, Tokyo University of Science (Shinjuku city, Tokyo, Japan). He is interested in coordination chemistry (hybrid systems of chiral Schiff base complexes and other functional materials), physical inorganic chemistry, crystallography.

Prof. Dr. Akitsu is a member of Chemical Society of Japan and some other professional societies and editorial board of some journals. He got Dr. Radhakanta Kajal Sen Merit Scholarship Award (4th World Science Congress) in 2014 at Jadavpur University, India. 